\title{
Urethral recurrence after radical cystectomy for urothelial carcinoma: a systematic review and meta-analysis
}

\begin{abstract}
Purpose: Currently, identified factors for urethral recurrence (UR) are based on individual reporting which has displayed controversy. In addition, risk of UR is one of the limiting factors to offer neobladder diversion during radical cystectomy (RC). We aim to systematically evaluate the incidence and risk factors of UR post-RC and its effect on survival. Materials and methods: A systematic online search was conducted according to PRISMA statement for publications reporting on UR after RC. From initial 802 results, 14 articles including 6169 patients were included finally after exclusion of ineligible studies. Results: The incidence rate of UR was $4.4 \%(1.3 \%-13.7 \%)$. It was significantly lower with neobladder diversion (odds ratio $=0.44,95 \% \mathrm{CI}: 0.24-0.79, \mathrm{P}=0.006$ ). Muscle invasion (hazard ratio $=1.18,95 \% \mathrm{CI}$ : 0.86-1.62, $\mathrm{P}=0.31$ ), carcinoma in situ (hazard ratio 0.97, 95\% CI: $0.64-1.47, \mathrm{P}=0.88$ ), prostatic stromal involvement (hazard ratio $=2.26,95 \% \mathrm{CI}: 0.01-627.75, \mathrm{P}=0.78$ ), and prostatic urethral involvement (hazard ratio $=2.04,95 \% \mathrm{CI}: 0.20-20.80, \mathrm{P}=0.55$ ) have no significant effect on UR. Men displayed tendency toward higher incidence of UR (odds ratio $=2.21,95 \%$ CI: 0.96-5.06, $\mathrm{P}=0.06$ ). Absence of recurrence displayed tendency toward better disease specific survival, yet not significant (hazard ratio $=0.84,95 \%$ CI: $0.66-1.08, \mathrm{P}=0.17$ ). These results are limited by the retrospective nature of the included studies. Conclusion: Muscle invasion, carcinoma in situ and prostatic stromal or urethral involvement at time of RC have no significant effect on UR. Orthotopic neobladder is associated with a significant lower risk of UR after RC.
\end{abstract}

Keyword: Bladder cancer; Neobladder; Radical cystectomy; Urethral recurrence; Urothelial carcinoma 